\title{
FORCE-DISTANCE SPECTROSCOPY: A GENERIC METHOD TO DETERMINE THE YOUNG'S MODULUS OF FREESTANDING NANOSTRUCTURES
}

\author{
N. Duarte ${ }^{1}$, Q. Xiong ${ }^{2,3}$, S. Tadigadapa ${ }^{1}$, P.C. Eklund ${ }^{2,3}$ \\ ${ }^{1}$ Department of Electrical Engineering, ${ }^{2}$ Department of Physics, ${ }^{3}$ Department of Materials Science and Engineering \\ The Pennsylvania State University, University Park, PA16802 United States
}

\begin{abstract}
:
We report here the determination of Young's modulus of bottom-up synthesized nanowires. AFM force-distance spectroscopy was performed at the mid-point of nanowire bridges fabricated using microfabrication techniques. The deflection per unit force was calculated from the difference in slope of the force distance curve on the nanowire bridge and a non-deformable surface. Young's modulus of the nanowires was subsequently deduced from the solution of the first mode of the Euler-Bernoulli beam equation with fixed-fixed boundary condition and a concentrated point load at the mid-point. The dimensions of the bridge were determined from atomic force microscopy (thickness) and field-emission scanning electron microscopy (length and width). We believe this method provides significant improvements on previously reported methods and can be used in any other nano-beam systems to measure their mechanical properties.
\end{abstract}

\section{Introduction:}

Understanding of mechanical properties of nanoscale materials, such as nanotubes or nanowires (NWs/NTs) is essential for their applications in nanomechanics ${ }^{1}$, NEMS devices ${ }^{2}$, sensors ${ }^{3}$, and nano- electronic ${ }^{4,5}$. Unfortunately many macroscopic techniques for measuring mechanical properties, e.g., tensile test, cannot be done routinely at nanoscale. As a result, nanomechanical measurements still remain a challenge and advances in reliable methods to perform these measurements are currently needed.

Atomic force microscopy (AFM) based techniques play a key role in such measurements. Wong et al. ${ }^{6}$ first reported elegant utilization of lateral force microscopy (LFM) to determine Young's modulus of single clamped non-suspended silicon carbide nanorods and multiwall carbon nanotubes (MWNTs). Song et al. ${ }^{7}$ also used LFM to measure the elastic modulus of vertically aligned $\mathrm{ZnO}$ nanowires. Due to the uncertainty in the lateral dimensions, length, and cross-section of the nanowires intrinsic to this method, the measured values of Young's modulus showed fairly large variation ${ }^{7}$. However, for vertically aligned nanostructures with short length and low density, this method can be a convenient approach. Salvetat et al showed that bending of MWNTs ${ }^{8}$ or single-walled carbon nanotubes (SWNTs) ropes ${ }^{9}$ could be measured under contactmode AFM, allowing the extrapolation of both elastic and shear moduli.

Recently, nanoindentation has emerged as an alternative technique to measure the Young's modulus and hardness of materials such as $\mathrm{ZnS}$ nanobelts ${ }^{10}$ and silver nanowires ${ }^{11}$. Nanoindentation is basically an AFM-based method, in which a diamond cantilever is used first as an imaging probe to locate the NWs, and then is used to indent the NWs in-situ. Force-displacement curves can be obtained for both load and unload situations and the morphology of the indent can be scanned sequentially. The hardness is given by analysis of the indentation and the Young's modulus is obtained from the unloading force-displacement curve ${ }^{11}$. The main benefits of this technique are that it does not require clamping on the ends of NWs and gives a quantitative measurement of hardness at nanoscale. In general however, it is not a satisfactory technique due to its destructive nature and the uncertainty in relating the sort of deformation (dislocation or plastic) occurring in the nanomaterials to the Young's modulus and hardness.

In this paper, we report a generic, AFM-based method to measure the Young's modulus of nanowires, using $\mathrm{ZnS}$ nanowires as an example. We have successfully fabricated clamped-clamped suspended nanobridges on $\mathrm{Si}$ substrates by standard microfabrication techniques. AFM was then used to locate the nanobridge and force-distance (FD) spectroscopy was performed at the middle point of the nanobridge. The slope of the FD curve obtained on the nanowire bridge can be compared with that obtained on non-deformable substrates to obtain the Young's modulus. The interaction between the nanobridge and the AFM tip was modeled by the Euler-Bernoulli beam equation with a fixed-fixed boundary condition and a concentrated point load. The Young's modulus of the nanowire was then deduced after an accurate determination of the bridge dimensions using AFM (thickness) and field-emission scanning electron microscopy (FESEM) (length and width). For the example of $\mathrm{ZnS}$ nanowires, the measured Young's modulus ranged from 45$65 \mathrm{GPa}$, which is about $10-20 \%$ smaller than that of the bulk. We believe this method can be used for any other nano-beam systems to measure their mechanical properties. $\mathrm{ZnS}$ nanowires were chosen as our study material because, as they have electroluminescent properties, they promise to be important in creating nanoeletronic and nano-optic devices ${ }^{13}$.

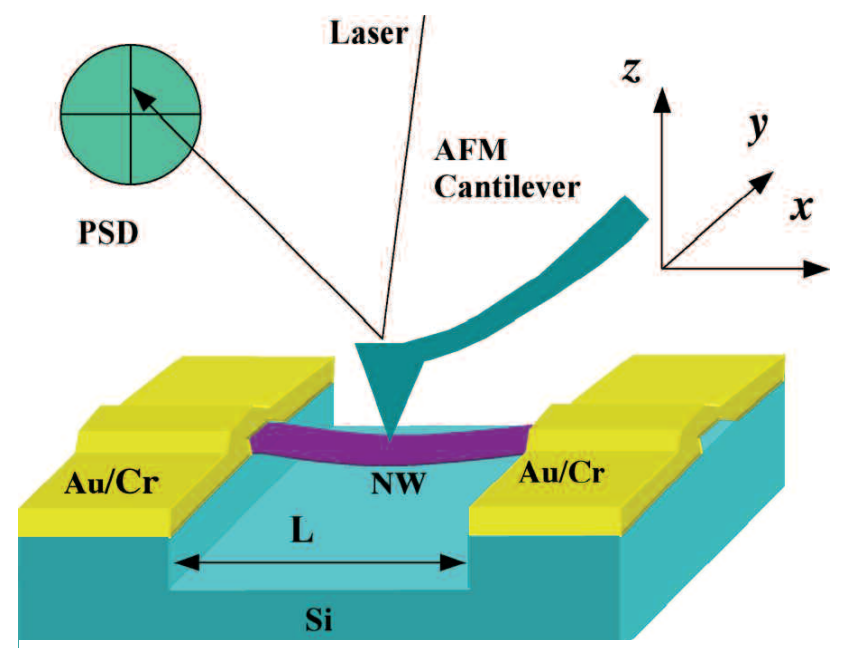

Figure 1: Schematic diagram of force-distance spectroscopy on a nanobridge. The nanobridge, which is fixed by $\mathrm{Au} / \mathrm{Cr}$ thin films at both ends. is across a trench in $\mathrm{Si}$ substrates.

\section{Fabrication:}

Nanowire nanobridges, shown schematically in Figure 1, were fabricated by dispensing bottom-up synthesized nanowires on a silicon wafer, followed by definition of endclamping pads by microfabrication and a final vapor phase $\mathrm{XeF}_{2}$ vapor phase release. The $\mathrm{ZnS}$ nanowires were grown by pulsed laser vaporization method ${ }^{12}$ and exhibit wurtzite structure with a rectangular cross section and lengths of more than ten microns. 




Figure 2: FE-SEM of an actual nanobridge

Details about growth and characterization of these nanowires with SEM, high-resolution TEM, and selective-area electron diffraction can be found elsewhere ${ }^{13,14}$. The as grown nanowires were then dispersed into ethanol by ultrasonication to obtain a metastable suspension. Several drops of this suspension were dispersed by spin coating onto a clean and native oxide removed silicon substrate. The Si substrate with $\mathrm{ZnS}$ nanowires was then dehydration baked for 5 minutes at $200^{\circ} \mathrm{C}$ on a hot plate. Lift-off photo resist LOR-1A (Microchem Corp.) was applied to the substrate and spun for 1 minute at 2,000 RPM, resulting in about $150 \mathrm{~nm}$ in thickness. A soft bake at $180^{\circ} \mathrm{C}$ for 5 minutes on the hot plate followed. Subsequently, photo resist SPR-3012 was applied to the substrate and spun for 1 minute at 4,000 RPM, resulting in a thickness of about $1,200 \mathrm{~nm}$. The wafer was soft baked at $100^{\circ} \mathrm{C}$ for 1 minute. After exposure and development, in MF-CD-26, the samples were transferred into an e-beam evaporator where $10 \mathrm{~nm} \mathrm{Cr}$ and $40 \mathrm{~nm} \mathrm{Au}$ films were deposited sequentially. Lift-off was carried out in $60^{\circ} \mathrm{C}$ bath of Remover PG (Microchem Corp.). Finally, $\mathrm{XeF}_{2}$ etching was carried out to release the nanowire and a trench with a depth of $\sim 500 \mathrm{~nm}$ in Si substrate was achieved. Figure 2 shows an FESEM (Leo 1530) image of the resulting nanobridge. The image shows that the etched surface in the trench is locally uniform and the bridge is clear of other materials. The depth of the trench is important since if the trench is too deep, the nanobridge may become unclamped. If the trench is too shallow, there might be some residual supporting pillars underneath the nanobridge, which will result in erroneous FD spectroscopy measurements. From our experiments, we believe $500-1000$

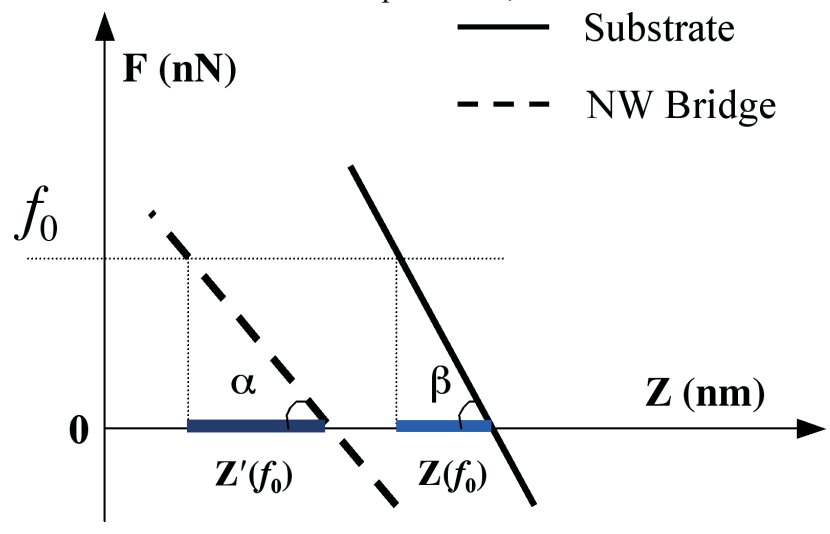

Fig.3: Ideal linear part of the force-distance (FD) spectra. $\mathrm{nm}$ depth is sufficient. Our device structure looks similar to a recent report on mechanical properties of Au nanowires ${ }^{15}$, however it should be noted that report used focused ion beam milling to define the trench, followed by deposition of the nanowires, AFM manipulation to position the nanowires, and ebeam induced deposition of platinum clamping pads. Our method is significantly simpler and provides higher yield as there is no risk of nanowires falling into the trenches during manipulation.

\section{Method:}

The principle of performing FD spectroscopy on a nanobridge is illustrated in Fig. 1. The nanobridge was first located under contact-mode AFM. Then the AFM cantilever, with a calibrated force constant of $0.13-0.17 \mathrm{~N} / \mathrm{m}$, was brought into contact with the middle point of the nanobridge where FD spectroscopy was performed. To avoid breaking the nanobridge, the amount of total force and Z-scanner range was limited.

Typical FD curves show the approaching (noncontact), jumping to contact due to van der Waals forces or capillary forces (snap-on), contact, adhesion and pull-off characteristic regimes ${ }^{16}$. For a hard and non-deformable sample, the slope of the FD curve in the contact regime gives the spring constant of the AFM cantilever. Figure 3 shows a schematic diagram of the linear part of FD curves obtained on a nondeformable substrate (solid line) and a deformable substrate, e.g., the nanobridge. The deflection of the nanobridge at a certain force is given by, $\Delta Z=Z^{\prime}\left(f_{0}\right)-Z\left(f_{0}\right)$ indicated on the horizontal axis.

For the nanobridge, due to its elastic compliance, the $Z$-scanner moves further for the same applied force. As a result, the FD curves obtained from a deformable substrate have smaller apparent slope, as indicated by dashed line. The deflection of the nanobridge at a certain force $f_{0}$ is given by:

$$
\Delta Z=Z^{\prime}\left(f_{0}\right)-Z\left(f_{0}\right)=\frac{f_{0}}{\tan \alpha}-\frac{f_{0}}{\tan \beta}
$$

where $\tan \alpha$ and $\tan \beta$ are the slopes of the FD curves in the linear contact regime. The force per unit deflection is:

$$
\frac{f_{0}}{\Delta Z}=\frac{\tan \alpha \tan \beta}{\tan \beta-\tan \alpha}
$$

The out-of-plane displacement $Z$ of the nanowire bridge, upon the application of the point load due to the AFM cantilever tip, can be characterized by the Euler-Bernoulli beam equation ${ }^{17}$ :

$$
E I \frac{d^{4} Z}{d x^{4}}=f \delta\left(x-x_{0}\right)
$$

where $E$ is the Young's modulus, $I$ is the area moment of inertia and $x_{0}$ is the position of the load. The coordinates have been defined in Figure 1. The assumption made is that $E$ and $I$ do not change along the length of the beam. The area moment of inertia, $I$, is $\pi d^{4} / 64$ for a cylindrical beam with a diameter of $d$, whereas for a rectangular beam with a width $w$ and a height $h$ $I=w h^{3} / 12{ }^{18}$. Using the fixed-fixed boundary condition, the solution to Eq. (3) is given by ${ }^{17}$ :

$$
Z=-\frac{f x^{2}}{48 E L}(3 L-4 x),(0 \leq x \leq L / 2)
$$

where $L$ is the total length of the nanobridge, which can be measured from AFM and FESEM. The negative sign means the deflection is downwards. At $x=L / 2$, the deflection $\Delta Z$ will be 


$$
\Delta Z=\frac{f L^{3}}{192 E I}
$$

Then the Young' modulus $E$ can be expressed as:

$$
E=\frac{f}{\Delta Z} \cdot \frac{L^{3}}{192 I}
$$

The first term in this formula is the force per unit deflection, measured by FD spectroscopy; while the second term is a geometrical factor associated with the dimension (i.e., length and the area moment of inertia) of the nanobridge. The geometrical factor can be determined by AFM and FESEM with good accuracy. For $\mathrm{ZnS}$ nanobridges with a rectangular cross section, eq. (6) can be specifically written as:

$$
E=\frac{f}{\Delta Z} \cdot \frac{L^{3}}{16 w h^{3}}=\frac{\tan \alpha \tan \beta}{\tan \beta-\tan \alpha} \cdot \frac{L^{3}}{16 w h^{3}}
$$

\section{Results:}

Figure 4 shows an AFM image taken at contact mode. Clear contrast of the trench and the nanobridge is observed. Figure 5 shows two typical FD curves obtained on substrate (blue curve) and the middle point of the nanobridge (red curve).

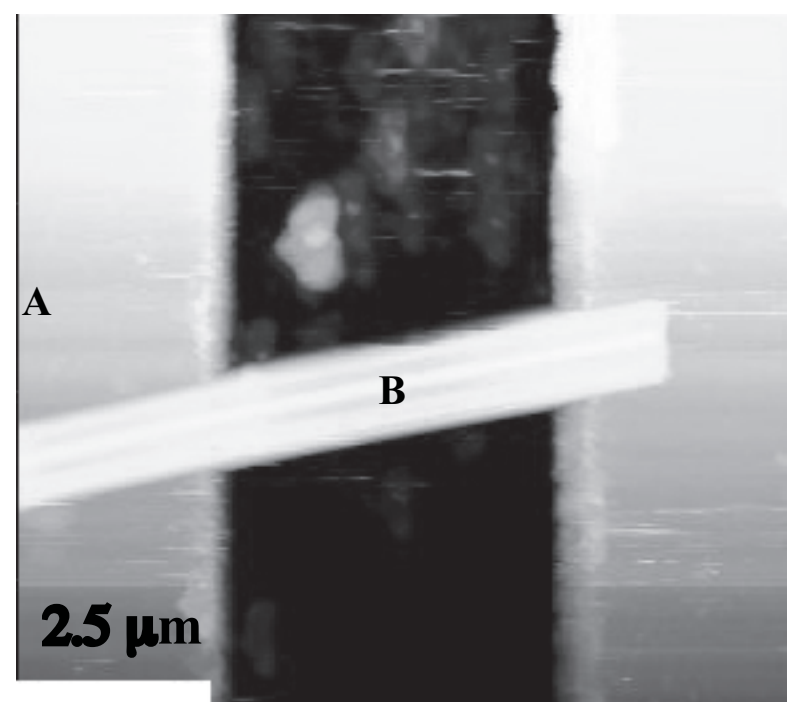

Fig.4: Contact mode AFM image of a $\mathrm{ZnS}$ nanobridge.

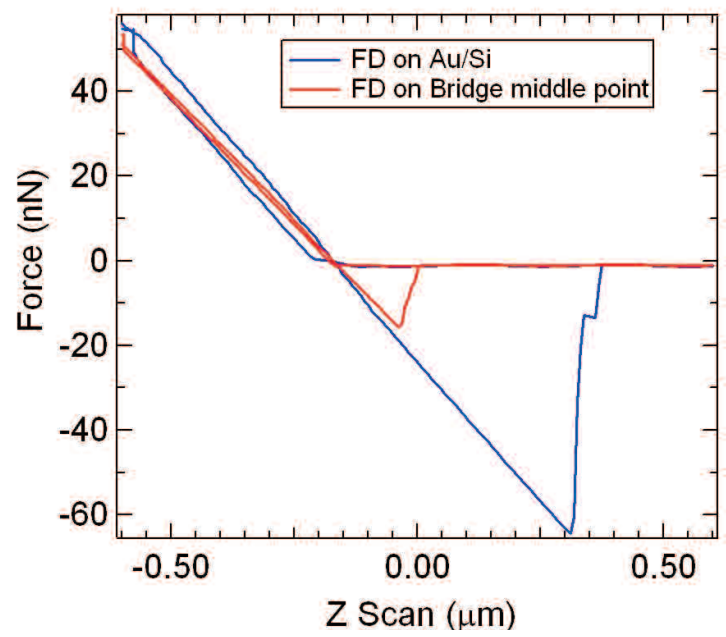

Fig.5: Force-distance curves obtained on the substrate for calibration (blue) and at the middle of the nanobridge (red).

\begin{tabular}{cccccc}
\hline $\begin{array}{c}\text { Sample } \\
\text { ID }\end{array}$ & $\begin{array}{c}\text { Geometric } \\
\text { Factor } \\
(1 / \mathrm{m})\end{array}$ & $\begin{array}{c}\tan \beta \\
(\mathrm{nN} / \mathrm{um})\end{array}$ & $\begin{array}{c}\tan \alpha \\
(\mathrm{nN} / \mu \mathrm{m})\end{array}$ & $\begin{array}{c}f / \Delta Z \\
(\mathrm{~N} / \mathrm{m})\end{array}$ & $\begin{array}{c}\text { Young's } \\
\text { Modulus } \\
(\mathrm{GPa})\end{array}$ \\
\hline A03 & $5.76 \mathrm{E}+10$ & 144 & 125 & 0.94 & 54.2 \\
$\mathrm{~A} 11$ & $5.40 \mathrm{E}+10$ & 137 & 119 & 0.93 & 50.4 \\
C1_02 & $1.70 \mathrm{E}+11$ & 148 & 106 & 0.38 & 64.1 \\
C2_01 & $2.30 \mathrm{E}+12$ & 143 & 20 & 0.03 & 51.8 \\
E1_01 & $5.81 \mathrm{E}+10$ & 155 & 129 & 0.77 & 44.9 \\
E2_01 & $3.84 \mathrm{E}+10$ & 162 & 142 & 1.13 & 43.2 \\
\hline
\end{tabular}

Table1: Tabulated results for several nanobridges. The geometric factor is from the second term in Eqn.(7).

The nanowire appears large in the AFM scan because of the tip size, as evidenced by the repeated structures in the trench. It is consistent with our above analysis on FD spectroscopy that the slope of the linear regime of the FD curve obtained on the nanobridge is lower in comparison with that obtained on the substrate. Least-square fitting was performed to extract the slope for both FD curves. Nanobridges with variable lengths $(\sim 2-10$ $\mu \mathrm{m})$ and different cross sectional dimensions have been measured. Table 1 lists all the results including the geometrical factor determined from AFM and FESEM, slopes for nanobridges and substrates, the calculated $f / \Delta Z$, and the calculated Young's modulus according to Eqn.(7). From Table 1 , we can see the measured Young's modulus is reasonably consistent. The overall average value is about $54.0 \pm 5.5 \mathrm{GPa}$, which is about $25 \%$ less than the bulk value of $75 \mathrm{GPa}$. This error value also indicates that our method more precise than previously reported methods which consistently have errors of 8 $\mathrm{GPa}$ or greater ${ }^{6,7}$. The only methods with better reported precision are nanoindentation ${ }^{10}(0.2-3.5 \mathrm{GPa})$ and lateral AFM bending ${ }^{10}$ (1.1-11 GPa), both of which are destructive methods.

It is worthwhile to point out that the adhesion force between $\mathrm{Au}$ film and $\mathrm{Si}$ is about $60 \mathrm{nN}$, much stronger than that between AFM cantilever and $\mathrm{ZnS}$ nanobridge. The adhesion forces between the AFM cantilever and the Au film are quite consistent while the adhesion forces between Si cantilever and the $\mathrm{ZnS}$ nanobridges varies from sample to sample $\sim 0-15 \mathrm{nN}$. In fact, some of the nanobridges even show negligible adhesion forces. This variation is probably due to the fact that the surface varies from wire to wire. HRTEM has shown that these $\mathrm{ZnS}$ nanowires have a very thin amorphous layer with unknown composition ${ }^{13,19}$. The small adhesion forces between AFM cantilever and $\mathrm{ZnS}$ nanobridge provide us two advantages: (i) The nanobridge will not be broken during approach of the AFM cantilever into contact with the bridge (snap-on), so FD spectroscopy can be non-destructively repeated; and (ii) The FD spectroscopy is still performed in the linear regime for the nanobridge. However, we do want to emphasize that the nanobridges are fragile and can be easily broken due to any carelessness during AFM operations. Slow scan rate and a slow scanning direction perpendicular to the nanobridge are required to minimize such accidental damage.

\section{Discussion:}

In contrast to our assumption of uniform cross-section for the nanowires, several reports have reported a modulation of the cross-sectional dimensions along the length of the nanowire axis 14,20 . This modulation actually leads to a symmetry breaking of the surface potential and therefore surface optic 
phonons are activated in Raman scattering $14,20$. Microscopically, in our FESEM and AFM images we have observed that the cross section (width in FESEM and height in AFM) of the nanobridge has some variations. Additionally we have observed a $5 \%$ variation from wire to wire. We think that this variation in width can explain the dispersion of the values of final Young's modulus. Additionally we are in the process of testing the precision of these measurements with multiple measurements on the same wires. With improvement of the geometrical factor, the accuracy of the Young's modulus measurement using this method can be improved.

It is still a matter of speculation whether the Young's modulus at nanoscale is different than that of bulk material, or at what scale the Young's modulus will be substantially different. It has been reported recently that the Young's modulus of $\mathrm{Au}$ nanowires $(40-250 \mathrm{~nm})$ is independent of diameter while the yield strength is diameter-dependent and is 100 times larger than that of the bulk materials ${ }^{15}$. On the other hand, it has also been reported that $1 \mathrm{D} \mathrm{Al}$ and $\mathrm{Au}$ nanostructures show a decrease in the Young's modulus in comparison with bulk state ${ }^{21}$. In semiconducting nanowires, the measured Young's modulus in LFM $^{7}$ and nanoindentation ${ }^{10}$ both show a reduction compared with that of the bulk materials. Our measured values of Young's modulus for $\mathrm{ZnS}$ nanowires are consistent with other reports on reduction of Young's modulus in the nanoscale ${ }^{7,10}$, showing a $10-20 \%$ reduction as compared to bulk values.

\section{Conclusion:}

We have used force-distance spectroscopy to study the mechanical properties of $\mathrm{ZnS}$ nanobridges, fabricated by standard microfabrication method. The interaction between the concentration forces exerted by AFM cantilevers and the nanobridge was modeled by the Euler- Bernoulli beam equation with a fix-end boundary condition. The Young's modulus was determined by a geometrical factor, which can be measured by AFM and FESEM, and a term of force per unit deflection, measured by force-distance spectroscopy. For $\mathrm{ZnS}$ nanowires, we measured the Young's modulus to be $51.5 \pm 7.5 \mathrm{GPa}$, which is a $30 \%$ decrease compared with bulk $\mathrm{ZnS}$. We believe this method based on force-distance spectroscopy and the model analysis can be used for other one-dimensional nano-beams down to $10 \mathrm{~nm}$ regime with the help of several steps of microfabrication.

\section{Acknowledgements:}

We thank Prof. E. Manias for discussion of forcedistance spectroscopy. The financial support was provided by NSF-NIRT (Nanotechnology and Interdisciplinary Research Initiative), Grant DMR-0304178.

\section{References:}

1. Kim, P.; Lieber, C. M., Nanotube nanotweezers. Science 1999, 286, (5447), 2148-2150.

2. Sazonova, V.; Yaish, Y.; Ustunel, H.; Roundy, D.; Arias, T. A.; McEuen, P. L., A tunable carbon nanotube electromechanical oscillator. Nature 2004, 431, (7006), 284 287.

3. Cui, Y.; Wei, Q. Q.; Park, H. K.; Lieber, C. M., Nanowire nanosensors for highly sensitive and selective detection of biological and chemical species. Science 2001, 293, (5533), 1289-1292.

4. Lieber, C. M., The incredible shrinking circuit Researchers have built nanotransistors and nanowires. Now they just need to find a way to put them all together. Scientific American 2001, 285, (3), 58-64.
5. Samuelson, L., Self-forming nanoscale devices. Materials Today 2003, 6, (10), 22-31.

6. Wong, E. W.; Sheehan, P. E.; Lieber, C. M., Nanobeam mechanics: Elasticity, strength, and toughness of nanorods and nanotubes. Science 1997, 277, (5334), 1971-1975.

7. Song, J. H.; Wang, X. D.; Riedo, E.; Wang, Z. L., Elastic property of vertically aligned nanowires. Nano Letters 2005, 5 , (10), 1954-1958.

8. Salvetat, J. P.; Kulik, A. J.; Bonard, J. M.; Briggs, G. A. D.; Stockli, T.; Metenier, K.; Bonnamy, S.; Beguin, F.; Burnham, N. A.; Forro, L., Elastic modulus of ordered and disordered multiwalled carbon nanotubes. Advanced Materials 1999, 11, (2), 161-165.

9. Salvetat, J. P.; Briggs, G. A. D.; Bonard, J. M.; Bacsa, R. R.; Kulik, A. J.; Stockli, T.; Burnham, N. A.; Forro, L., Elastic and shear moduli of single-walled carbon nanotube ropes. Physical Review Letters 1999, 82, (5), 944-947.

10. Li, X. D.; Wang, X. N.; Xiong, Q.; Eklund, P. C., Mechanical properties of $\mathrm{ZnS}$ nanobelts. Nano Letters 2005, 5, (10), 1982-1986.

11. Li, X. D.; Hao, H. S.; Murphy, C. J.; Caswell, K. K., Nanoindentation of silver nanowires. Nano Letters 2003, 3, (11), 1495-1498.

12. Morales, A. M.; Lieber, C. M., A laser ablation method for the synthesis of crystalline semiconductor nanowires. Science 1998, 279, (5348), 208-211.

13. Xiong, Q.; Chen, G.; Accord, J. D.; Liu, X.; Gutierrez, H.; Redwing, J. M.; Lew Yan Voon, L. C.; Lassen, B.; Eklund, P. C., Optical properties of rectangular cross-sectional $\mathrm{ZnS}$ nanowires. Nano Letters 2004, 4, (9), 1663-1668.

14. Xiong, Q.; Wang, J. G.; Reese, O.; Voon, L. C. L. Y.; Eklund, P. C., Raman scattering from surface phonons in rectangular cross-sectional w-ZnS nanowires. Nano Letters 2004, 4, (10), 1991-1996.

15. Wu, B.; Heidelberg, A.; Boland, J. J., Mechanical properties of ultrahigh-strength gold nanowires. Nature Materials 2005, 4, (7), 525-529.

16. Prater, C. B.; Maivald, P. G.; Kjoller, K. J.; Heaton, M. G., Probing nano-scale forces with the atomic force microscope. Veeco Metrology Group.

17. Gere, J. M.; Timoshenko, S. P., Mechanics of materials 4th ed.; PWS Pub Co.: Boston, 1997; p xvi, 912.

18. Young, W. C.; Budynas, R. G., Roark's formulas for stress and strain. 7th ed.; McGraw-Hill: 2000.

19. Xiong, Q.; Gupta, R.; Adu, K. W.; Dickey, E. C.; Lian, G. D.; Tham, D.; Fischer, J. E.; Eklund, P. C., Raman spectroscopy and structure of crystalline gallium phosphide nanowires. Journal of Nanoscience and Nanotechnology 2003, 3, (4), 335339.

20. Gupta, R.; Xiong, Q.; Mahan, G. D.; Eklund, P. C., Surface optical phonons in gallium phosphide nanowires. Nano Letters 2003, 3, (12), 1745-1750.

21. Haque, M. A.; Saif, M. T. A., Deformation mechanisms in free-standing nanoscale thin films: A quantitative in situ transmission electron microscope study. Proceedings of the National Academy of Sciences of the United States of America 2004, 101, (17), 6335-6340.

22. Read, A. J. N., R. J.; Nash, K. J.; Canham, L. T.; Calcott, P. D.; A. Qteish, First-principle calculation of the electronic properties of the silicon quantum wires. Physical Review Letters 1992, 69, (8), 1232-1235. 